\title{
Ambientes pastoriles de un establecimiento foresto-ganadero de la localidad de Chavarría (Corrientes).
}

\author{
Castelan $^{1}$, María E.; Porta, Miriam; Hack, Claudina M.; Salvia, María. T.; Ciotti ${ }^{2}$, Elsa M. \\ Instituto Agrotécnico "Pedro M. Fuentes Godo", FCA-UNNE. Cátedra Forrajicultura, FCA-UNNE \\ Las Heras 727, Resistencia, Chaco
}

\begin{abstract}
Resumen
El objetivo del presente trabajo fue establecer la condición de ambientes pastoriles de la Localidad de Chavarría - Corrientes. El establecimiento Toro Cuaré está situado en el Departamento San Roque, provincia de Corrientes, localidad de Chavarría. El ecosistema predominante en el área de estudio es de lomadas y planicies arenosas. Se realizaron censos para determinar la composición florística de la comunidad vegetal. Para establecer la homogeneidad del pastizal se determinó la cobertura de cada especie expresada en porcentaje. También se agruparon las especies según la categorización en pastos ordinarios, duros, finos y tiernos. Se determinó la producción de forraje disponible, mediante el método de corte y pesada en 12 jaulas de exclusión del pastoreo. Para la caracterización de los ambientes se utilizó una metodología que considera tanto aspectos cualitativos como cuantitativos propuesto por Martín (2004). En el área de estudio se consideraron los valores de la comunidad Espartillar o pastizal. Para el bajo se tomaron como referencia los de la comunidad hidrófila ó praderas húmedas. Los mayores valores de presencia y cobertura corresponden a Andropogon lateralis Nees, Cyperus rotundus y Axonopus argentinus Parodi. En el sitio de estudio los pastos dominantes son incluidos dentro de la clasificación de ordinarios (A. lateralis) y duros (Cyperus spp.). Los pastos finos y los tiernos, como Axonopus argentinus y Paspalum spp., alcanzan un 30\% de cobertura. El rendimiento de materia seca obtenido en el pastizal fue de 2498 y $867 \mathrm{Kg} \mathrm{MS} \mathrm{ha}^{-1}$ en verano y otoño respectivamente. En la comunidad hidrófila en verano alcanzó los 2760 y en otoño $1850 \mathrm{Kg} \mathrm{MS} \mathrm{ha}^{-1}$. La condición del pastizal es muy buena y buena para la comunidad hidrófila o pradera húmeda.
\end{abstract}

Palabras clave: pastizal, caracterización, condición

\begin{abstract}
Summary
The aim of this work was to determine Chavarria (Corrientes) grasslands condition. The Toro Cuaré farm is located in Chavarria, San Roque Department, Corrientes Province. The predominant ecosystem is characterized by sandy hills and plains. A vegetation survey was done to determine the floristic composition of the community. The vegetation homogeneity was evaluated by the percentage of coverage of each species. Species were assigned to one of these groups: ordinary, coarse, fine and tender grasses. Available forage yield was determined using the cutting - weighing method using 12 grazing exclusion cages. Martin methodology (2004) was used for environment characterization. Values of Elionurus muticus comunity or grassland were considered in the area of study. For the lowlands, hydrophyte community or humid prairies were used as reference. The highest values of presence and coverage were obtained for Andropogon lateralis Nees, Cyperus rotundus y Axonopus argentinus Parodi. In the area, predominant grasses belong to ordinary (A .lateralis ) and rough (Cyperus spp.) category. Fine and tender grasses, such as Axonopus argentinus and Paspalum spp. reached up to $30 \%$ coverage. Dry matter yield was 2498 and $867 \mathrm{Kg} \mathrm{DM} \mathrm{ha}^{-1}$ in summer and autumn, respectively. In the hydrophyte community, yield was $2760 \mathrm{Kg} \mathrm{DM} \mathrm{ha}^{-1}$ in summer and $1850 \mathrm{Kg} \mathrm{DM} \mathrm{ha}^{-1}$ in autumn. Grassland condition was very good, whereas hydrophyte and prairie community condition were good. Key words: Grassland, characterization, condition
\end{abstract}

\section{INTRODUCCIÓN}

Los pastizales del Río de la Plata son parte de la unidad biogeográfica de pastizales más extensa de Sudamérica y una de las más importantes del mundo. Esta unidad ocupa un área de 70 millones de ha y abarca el este de Argentina, Uruguay y Río Grande del Sur en Brasil (Soriano, 1991). La ganadería vacuna y ovina es su principal actividad económica.
Dentro de esta gran región, el NEA comprende subregiones ambientalmente muy diferentes, teniendo en cuenta que las precipitaciones varían desde 500 mm en el Oeste del Chaco y $2000 \mathrm{~mm}$ en el Este de Misiones. Además hay una gran diversidad de suelos, topografía, vegetación y, en consecuencia, distinta aptitud productiva (Jacobo y Rodríguez, 2009). 
En la Región Occidental de la provincia de Corrientes en el área de Lomadas Arenosas, Planicies y Depresiones se encuentran pastizales de Andropogon lateralis (paja colorada), Sorghastrum setosum (paja amarilla) y Elionurus muticus (espartillo) (Carnevali, 1994). Estos se desarrollan en ambientes de alta pluviometría y productividad potencial. Presentan un gran desarrollo en altura, con dominancia de una a dos especies y otras especies acompañantes, que crecen activamente desde la primavera hasta el otoño y son capaces de mantener el suelo cubierto durante todo el año con una alta proporción de biomasa seca en pie (Morello y Adámoli, 1968). La producción de la biomasa aérea acumulada varía según sitios y años (Gándara, 2003).

En el NEA, el sistema de pastoreo predominante es el continuo, caracterizado por tener una alta frecuencia de defoliación y una intensidad de pastoreo dependiente de la carga animal (Pizzio, 1995). Existe una gran selectividad de las especies más palatables, que como consecuencia se deterioran y disminuyen su aporte, favoreciendo el avance y el establecimiento de otras especies de menor calidad. Con este sistema se produce una degradación paulatina del suelo y la vegetación, con la consiguiente pérdida de productividad (Luisoni y Bissio, 1993; De León, 2003). Puede decirse que el manejo del pastoreo es un factor crítico en el mantenimiento de los pastizales. El desafío es utilizar los pastizales de manera sostenible ya que la aptitud natural de la región está determinada por el complejo climático-edáfico-forrajero. La clave del manejo del campo natural es el conocimiento de las bondades y limitaciones del recurso forrajero. Para tomar decisiones adecuadas sobre el manejo es necesario caracterizar previamente el pastizal (Martin, 2004).

El objetivo del presente trabajo fue caracterizar los ambientes pastoriles de un establecimiento foresto-ganadero ubicado en el departamento San Roque.

\section{MATERIALES Y MÉTODOS}

\section{Descripción general del área de estudio}

El establecimiento Toro Cuaré está situado en el departamento San Roque, provincia de Corrientes a $28^{\circ} 44^{\prime}$ S y $58^{\circ} 19^{\prime}$ O y a $60 \mathrm{msnm}$. Consta de 35000 ha, principalmente destinadas a la forestación con $P i$ nus ellioti y Pinus taeda. Para la actividad ganadera se utilizan 3000 ha, con una carga promedio de 0,5 $\mathrm{EV} \mathrm{ha}^{-1}$. Las categorías que integran el rodeo se manejan juntas, cambiándose de potrero una vez que la disponibilidad de forraje disminuye. El tiempo promedio de pastoreo es de 110 días en cada potrero.

Los datos de precipitación, temperatura, humedad, vientos y nubosidad fueron suministrados por E.V.A.S.A (Empresas Verdes S.A.), ya que el esta- blecimiento cuenta con una estación meteorológica automatizada.

Se consideran los datos de precipitación y temperaturas medias mensuales durante el período de evaluación (Figura 1).

El ecosistema predominante en el área de estudio es de lomadas y planicies arenosas. Se observa un relieve suavemente ondulado con lagunas circulares y semicirculares (Carnevali, 1994). Los suelos corresponden a las series Pampín y Chavarría que caracterizan la zona de lomadas arenosas del centro-oeste de la provincia. Básicamente son suelos de muy baja fertilidad y de textura dominante arenosa-franco en superficie (INTA, 1990).

\section{Evaluaciones}

Se seleccionó un área homogénea y representativa del lugar, donde se llevaron a cabo las evaluaciones correspondientes para determinar la condición actual del pastizal, a través de una recorrida de reconocimiento del establecimiento y con el apoyo de imágenes satelitales.

Se realizaron censos para determinar la composición florística de la comunidad vegetal, en los meses de marzo y junio. Para ello se trazaron dos transectas paralelas, de cincuenta metros cada una. Cada cinco metros se colocaron marcos de madera de $0,5 \mathrm{~m}$ de lado, registrando las principales especies de valor forrajero nativas o naturalizadas y sus valores de abundancia - dominancia de acuerdo a la escala del método de Braun Blanquet (1979). Se calculó el porcentaje de presencia de cada especie. Para establecer la homogeneidad del pastizal se determinó la cobertura de cada especie expresada en porcentaje (Martín, 2004). También se agruparon las especies según la categorización de Rosengurt (1979) en pastos ordinarios, duros, finos y tiernos.

Se determinó la producción de forraje disponible, mediante el método de corte y pesada. El 21 de diciembre de 2010 se instalaron al azar 12 jaulas de exclusión del pastoreo para evitar que los animales consuman el rebrote. Las jaulas, de $1 \mathrm{~m}^{2}$ cada una, se

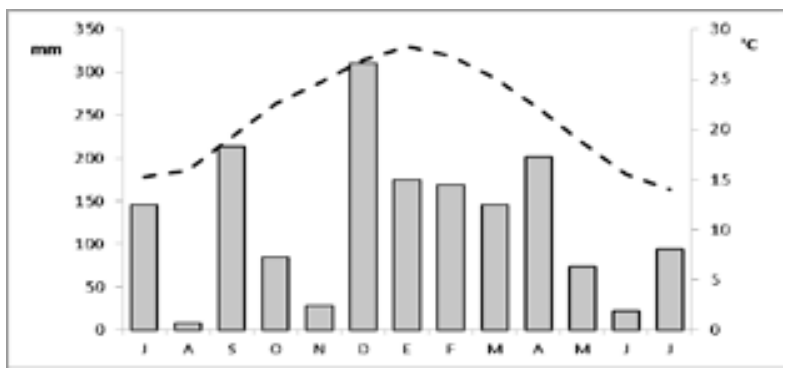

Figura 1. Precipitaciones $(\mathrm{mm})$ y temperaturas medias $\left({ }^{\circ} \mathrm{C}\right)$ mensuales durante el periodo de evaluación (Julio 2010-2011) 
Tabla 1. Escala de valores para la ponderación de cada atributo evaluado en la caracterización de un pastizal

\begin{tabular}{|c|c|c|}
\hline Atributo & Ponderación & Característica \\
\hline \multicolumn{3}{|l|}{ 1- Organización espacial de las especies } \\
\hline \multirow{5}{*}{$\begin{array}{l}\text { Los individuos en una comunidad no } \\
\text { se distribuyen aleatoriamente, forman } \\
\text { colonias más o menos grandes. }\end{array}$} & 5 & $\begin{array}{l}\text { Especies reunidas en formaciones generalmente amplias } \\
\text { en el espacio }\end{array}$ \\
\hline & 4 & Especies reunidas en pequeñas colonias \\
\hline & 3 & Especies reunidas en haces \\
\hline & 2 & Especies reunidas en grupos \\
\hline & 1 & Especies aisladas \\
\hline \multicolumn{3}{|l|}{ 2- Homogeneidad del pastizal } \\
\hline \multirow{4}{*}{$\begin{array}{l}\text { Presencia en porcentaje de la } \\
\text { abundancia de los grupos de especies } \\
\text { que definen la comunidad, en cada sitio } \\
\text { muestreado. }\end{array}$} & 4 & $80-100 \%$ \\
\hline & 3 & $75 \%$ \\
\hline & 2 & $50 \%$ \\
\hline & 1 & $<=25 \%$ \\
\hline \multicolumn{3}{|l|}{ 3- Vitalidad. } \\
\hline \multirow{4}{*}{$\begin{array}{l}\text { Característica que se refiere } \\
\text { al crecimiento de las especies } \\
\text { predominantes }\end{array}$} & 4 & Vigorosa (gran número de macollos o tallos) \\
\hline & 3 & Normal \\
\hline & 2 & Débil \\
\hline & 1 & Muy débil ( hay plantas muertas y/o marchitas) \\
\hline \multicolumn{3}{|l|}{ 4- Suelo } \\
\hline \multirow[t]{4}{*}{ 4-1 Pendiente } & 4 & Área plana $(<0.2 \%)$ \\
\hline & 3 & Área suavemente ondulada $(0,2 \%-0.4 \%)$ \\
\hline & 2 & Área moderadamente ondulada o inclinada $(0,4 \%-0,6 \%)$ \\
\hline & 1 & Áreas inclinada $(>0,6 \%)$ \\
\hline \multirow[t]{4}{*}{ 4-2 Compactación superficial } & 4 & No hay signos \\
\hline & 3 & $\begin{array}{l}\text { Estructura superficial laminar, sectores con costras } \\
\text { ( } \leq 30 \% \text { de la superficie diagnosticada) }\end{array}$ \\
\hline & 2 & $\begin{array}{l}\text { Estructura superficial laminar, sectores con costras } \\
\text { ( }>30 \% \text { y } \leq 60 \% \text { de la superficie diagnosticada) }\end{array}$ \\
\hline & 1 & $\begin{array}{l}\text { Sectores totalmente compactados, a más del } 60 \% \text { de la } \\
\text { superficie diagnosticada }\end{array}$ \\
\hline \multirow[t]{4}{*}{ 4-3 Encharcamiento } & 4 & $\begin{array}{l}\text { Sin encharcamiento: infiltración rápida y/o escurrimiento } \\
\text { superficial rápido }\end{array}$ \\
\hline & 3 & Encharcamiento uniforme y temporario \\
\hline & 2 & $\begin{array}{l}\text { Encharcamiento con escurrimiento laminar lento y } \\
\text { uniforme }\end{array}$ \\
\hline & 1 & Saturación del suelo y anegamiento permanente \\
\hline \multirow[t]{3}{*}{ 4-4 Presencia de sales en superficie } & 3 & Sin presencia \\
\hline & 2 & Por manchones, sectorizado ( $\leq 50 \%)$ \\
\hline & 1 & Grandes sectores ( $\geq 50 \%$ ) \\
\hline \multirow{5}{*}{$\begin{array}{l}\text { 4-5 Porcentaje de suelo cubierto por } \\
\text { broza }\end{array}$} & 5 & $>$ del $75 \%$ \\
\hline & 4 & $50-75 \%$ \\
\hline & 3 & $25-50 \%$ \\
\hline & 2 & $<25 \%$ \\
\hline & 1 & sin broza \\
\hline
\end{tabular}

distribuyeron siguiendo el gradiente de pendiente del terreno (loma, media loma, bajo). Dentro de las jaulas se efectuó un corte de emparejamiento, sin recolección del material, con el fin de homogeneizar la altura. Luego se realizaron cortes a los 91(verano) y 75 días (otoño). La altura de corte fue a $10 \mathrm{~cm}$, teniendo en cuenta la altura de pastoreo del ganado vacuno. Las muestras se secaron en estufa a $60^{\circ} \mathrm{C}$ hasta peso constante y se determinó la producción de materia seca (MS).

\section{Caracterización del pastizal}

Para caracterizar el pastizal se utilizó una metodología que considera tanto aspectos cualitativos como cuantitativos del pastizal (Martin, 2004). Las escalas 
Tabla 2. Escala de condición para cada comunidad (Martin, 2004)

\begin{tabular}{lccccc}
\hline \multirow{2}{*}{ Comunidad } & \multirow{2}{*}{ Tipo } & Bueno & Regular & Malo \\
\cline { 2 - 6 } & & Muy Bueno & 26 a 32 & 19 a 25 & $<19$ \\
\hline Flechillares & $(1 a, 1 b$ y 1c $)$ & 33 & 18 a 21 & 13 a 17 & $<13$ \\
Comunidad Halófila & $(2 a$ y 2b) & 22 & 25 a 28 & 17 a 25 & $<17$ \\
Comunidad Hidrófila & 4 & 29 & 17 a 18 & 15 a 16 & $<15$ \\
Espartillares & 3 & 19 &
\end{tabular}

para ponderar los atributos de vegetación y suelo utilizados en esta metodología se consignan en la tabla 1.

\section{Escala de condición del pastizal}

Sumando todas las variables ponderadas y en función del tipo de comunidad es posible inferir la condición del pastizal, empleando para ello una escala (Tabla 2)

En el área de estudio se consideraron los valores de la comunidad Espartillar o pastizal para la loma y media loma. Para el bajo se tomaron como referencia los de la comunidad hidrófila ó praderas húmedas.

\section{RESULTADOS Y DISCUSIÓN}

Dentro del área de estudio la cobertura vegetal fue del $100 \%$. Se encontraron especies forrajeras que pertenecen principalmente a la familia de Gramíneas y Ciperáceas. Las malezas son de distintas familias, siendo la mayoría Compuestas. Tanto en la loma como en la media loma del pastizal se observó también la presencia de especies de la familia Leguminosas como Desmodium barbatum, Adesmia bicolor e Indigofera suffruticosa con muy baja cobertura.

La zona de lomas tiene una pendiente casi nula y no se registran encharcamientos. Las especies del tapiz son pastoreadas a una altura entre 5 y 15 centímetros, incluso los rebrotes de paja colorada y paja amarilla (A. lateralis y $S$. setosum). Cuando estas especies están en prefloración y floración ya no son consumidas por el ganado, alcanzando $160 \mathrm{~cm}$ de altura. En zonas bajas, con agua permanente, cabe mencionar la presencia de Ciperáceas de porte alto,

Tabla 3. Presencia y cobertura (\%) de las especies encontradas

\begin{tabular}{lcc}
\hline Especie & $\begin{array}{c}\text { Presencia } \\
\%\end{array}$ & $\begin{array}{c}\text { Cobertura } \\
\%\end{array}$ \\
\hline Axonopus argentinus & 65 & 24 \\
Andropogon lateralis & 52 & 40 \\
Cyperus rotundus & 67 & 37 \\
Leersia hexandra & 25 & 5 \\
Rynchospora praecincta & 15 & 3 \\
Panicum miliaceum & 17 & 1 \\
Paspalum almum & 15 & 1 \\
Paspalum modestum & 10 & 1 \\
Broza & 50 & 10 \\
\hline
\end{tabular}

mientras que, en áreas con período de inundación temporario se encontraron especies como Hymenachne amplexicaule, Paspalum modestum y Paspalum almum, sin presencia de Leguminosas.

De acuerdo a los censos, las especies con mayor porcentaje de presencia y cobertura son A. lateralis, A. argentinus y Cyperus rotundus. En el resto de las especies los valores de presencia varían entre $10 \mathrm{y}$ $25 \%$ pero con muy baja cobertura, de 1 a $5 \%$ (Tabla $3)$.

Hack et al. (2009) determinaron la composición botánica de un pastizal de la misma zona ecológica, que tenía 58\% de Gramíneas, 22\% de Leguminosas, $10 \%$ de Ciperáceas y el 10\% restante a otras familias botánicas.

En el pastizal analizado en este estudio la predominancia de Gramíneas alcanza el $72 \%$, las Ciperáceas el 18\%, Leguminosas el $6 \%$ y otras familias el $4 \%$.

En el sitio de estudio los pastos dominantes son incluidos dentro de la clasificación de ordinarios ( $A$. lateralis) y duros (Cyperus spp.). Los pastos finos y los tiernos, como Axonopus argentinus y Paspalum almum, alcanzan un $30 \%$ de cobertura. Estos han sido reportados como los más apetecibles (Rosengurtt, 1979) (Figura 2).

Los tipos productivos se identifican como pasto fino de productividad alta o media y aceptabilidad prolongada; pasto tierno de productividad alta o media y aceptabilidad prolongada a media; pasto ordinario que tiene baja aceptabilidad, limitada generalmente al estado más o menos juvenil con una pro-

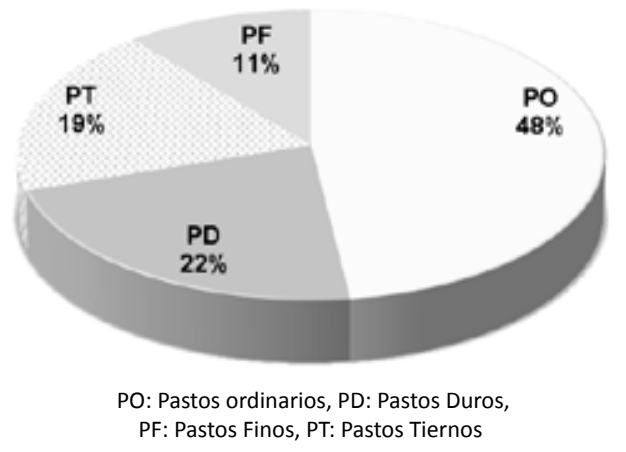

Figura 2. Distribución en porcentaje de las especies agrupadas por su valor forrajero. 
Tabla 4. Ponderación de los atributos en dos ambientes: Pastizal y Comunidad hidrófila y determinación de la condición.

\begin{tabular}{lcc}
\hline \multirow{2}{*}{ ATRIBUTO } & \multicolumn{2}{c}{ PONDERACION } \\
\cline { 2 - 3 } & Pastizal & $\begin{array}{c}\text { Comunidades } \\
\text { Hidrófilas }\end{array}$ \\
\hline Organización espacial de las especies & 5 & 4 \\
Homogeneidad & 3 & 3 \\
Vitalidad & 3 & 3 \\
Pendiente & 4 & 4 \\
Compactación superficial & 4 & 4 \\
Encharcamiento & 4 & 1 \\
Presencia de sales & 3 & 3 \\
Porcentaje de suelo cubierto por broza & 3 & 4 \\
\hline Total & 30 & 27 \\
\hline Condición & Muy Bueno & Bueno \\
\hline
\end{tabular}

ralis, cuya producción en esas estaciones del año alcanzó valores de 2374 y de 1142 $\mathrm{kg}$ de MS ha ${ }^{-1}$ respectivamente.

En el pastizal el $60 \%$ del rendimiento es aportado por las especies de porte alto como A. lateralis y de porte bajo como $A$. argentinus, $P$. almum. Mientras que en la pradera húmeda lo hacen las especies de la familia Ciperáceas como Rhynchospora praecincta (pirí) y las gramíneas llamadas "pastitos de agua" (Leersia hexandra Sw y Luziola peruviana Juss. ex. J.F. Gmel.) y especies del género Paspalum.

Los resultados obtenidos muestran que la condición del pastizal es muy buena y buena para la comunidad hidrófila o pradera húmeda, indicando que con la actividad ganadera actual, no existen indicios

Tabla 5. Rendimiento de materia seca (Kg MS ha $\left.{ }^{-1}\right)$ obtenido en cada ambiente

\begin{tabular}{lcc}
\hline & \multicolumn{2}{c}{ kg MS.ha $^{-1}$} \\
\cline { 2 - 3 } & Verano & Otoño \\
\hline Pastizal & 2498 & 867 \\
Comunidades Hidrófilas & 2760 & 1850 \\
\hline
\end{tabular}

ductividad mínima, los pastos duros tienen un porte elevado sobre el tapiz y su aceptabilidad es reducida al período juvenil de cada hoja, tienen una productividad mayor que las ordinarias. Las malezas tienen nula aceptabilidad (Rosengurtt; 1979).

\section{Condición del pastizal}

A cada atributo de los ambientes en estudio se asignó un valor de la escala y con ellos se determinó la condición. En la tabla 4 se detallan los resultados.

La condición del pastizal está considerada muy buena para el pastizal y buena para las comunidades hidrófilas o praderas húmedas (Tabla 4). La composición de especies de la comunidad vegetal no ha variado respecto de la vegetación descripta para esa zona (Carnevali, 1994). De acuerdo a la clasificación de Rosengurt (1979) se observó que predominan las especies deseables e intermedias, vigorosas y moderadamente productivas y existen unas pocas especies invasoras. El suelo tiene cobertura vegetal, lo que evita su erosión, facilita la retención de agua y su aireación, que se traduce en una mayor producción de forraje.

\section{Producción de materia seca}

Los resultados obtenidos en el rendimiento de MS en el presente ciclo de evaluación (Tabla 5) son similares en el verano y menores en otoño a los mencionados por Hack et al. (2009) en un pastizal de A. late- de que se encuentren en riesgo la productividad ni la persistencia de especies valiosas de esos ambientes.

Cingolani et al. (2008) menciona a la carga ganadera de un área como el principal factor que influye sobre su biodiversidad, suelo y producción. Por ello, en el sitio estudiado sería conveniente seguir con las pautas generales de manejo, basadas fundamentalmente en un sistema de pastoreo con descansos y carga animal de acuerdo a la receptividad de cada potrero, con el fin de preservar las especies de valor forrajero, lograr una conversión efectiva del forraje en producción animal y mantener a través del tiempo la producción y condición del pastizal.

\section{BIBLIOGRAFÍA}

Braun Blanquet J.; 1979. "Fitosociología: bases para el estudio de las comunidades vegetales". H. Blume Ediciones, Madrid.

Carnevali, R. 1994. Fitogeografía de la Provincia de Corrientes. Ed. Gobierno de la Provincia de Corrientes. Instituto Nacional de Tecnología Agropecuaria.

Cingolani, A. M.; Noy-Meir, I.; Renison, D. D. \& Cabido, M. 2008. La ganadería extensiva, ¿es compatible con la conservación de la biodiversidad y de los suelos? Ecología Austral 18:253-271.

De León, M. 2003. El Manejo de los Pastizales Naturales. Boletín Técnico Producción Animal, EEA Manfredi, Año I, No 2 y 3.

Gándara, F.R. 2003. "Manejo de Campo Natural". Misceláneas Centro Regional Chaco Formosa.

Hack, C.M., Tomei, C.E., Ciotti, E.M. y Castelán, M.E. 2009. Producción de un pastizal del SO de corrientes bajo condiciones de pastoreo controladas. $27^{\circ} \mathrm{Con}-$ greso Argentino de Producción Animal.

I.N.T.A - S.A.G.y.P.; 1990. Atlas de suelos de la República Argentina. Instituto Nacional de Tecnología Agropecuaria - Secretaría de Agricultura, Ganadería y Pesca, Buenos Aires- Argentina. 
Jacobo, E. y Rodríguez, A. 2009. Valorización de pastizales naturales en ambientes húmedos. Indicadores de sustentabilidad. 5to Congreso de la Asociación Argentina para el Manejo de Pastizales Naturales.

Luisoni, L.H. y Bissio, J.C. 1993. Unidades de vegetación y condición ganadera en un área de la cuña boscosa santafesina. INTA EEA Reconquista. Publicación Técnica $\mathrm{N}^{\circ} 7$.

Martín, B. 2004. "Metodología para la caracterización de pastizales naturales". Rev. Agromensajes. Universidad Nacional de Rosario, Santa Fe.

Morello, J. y Adámoli, J. 1968. Las grandes unidades de vegetación y ambiente del Chaco Argentino II. INTA, Serie Fitogeográfica (10), 1-126.

Pizzio, R M. 1995. Manejo de los campos naturales de la región este de la provincia de Corrientes. Jornadas internacionales de actualización ganadera subtropical: $67-75$.
Rosengurtt, B. 1979. “Tablas de comportamiento de las especies de plantas de campos naturales en el Uruguay”. Fac. Agr. Univ. de la Rep. Montevideo. Uruguay.

Soriano, A. 1991. Río de la Plata Grasslands. En: Jacobo, E. y Rodríguez, A. Valorización de pastizales naturales en ambientes húmedos. Indicadores de sustentabilidad. 5 Congreso de la Asociación Argentina para el Manejo de Pastizales Naturales, 2009.

Zuloaga, F.O.; O., Morrone \& M.J., Belgrano 1994 en adelante. Catálogo de Plantas Vasculares del Cono Sur. Website http://www.darwin.edu.ar/Proyectos/ FloraArgentina/FA.asp. Actualizado a Enero 2009. Consulta: abril 2012. 